\title{
Yarn Tension in Winding Mechanism.
}

\author{
Part 1: Behavior of Tension in a Running Yarn. \\ By Tatsuo Nakashima, Kyuya Takagi and Takizo Aoyama, Members, TMSJ \\ Nagoya Municipal Industrial Research Institute, Nagoya \\ Based on the Journal of the Textile Machinery Society of Japan, Transactions, Vol. 19, No. 5, T131-141(1966)
}

\begin{abstract}
The responses of tension in a running yarn given stepwise friction at two points and drafted stepwise at two other points in the winding system have been studied with the following results:

Assuming the yarn to be perfectly elastic, the tension is readily calculable and agrees well with experimental results on nylon and cotton yarn. To make a more exact estimate, the effects of tension "Career" and creep of the yarn must be taken into consideration. "Career" means the amount of tension a sample received being used in our experiments.
\end{abstract}

\section{Introduction}

Control of the tension of a running yarn in the winding, warping, sizing, weaving and other process is highly important. It is a difficult task, however, because a yarn, a complex fiber assembly, is neither perfectly elastic nor simply visco-elastic. With friction, draft and other factors acting on a running yarn tension shows a complex behavior and cannot be kept constant. Accordingly, any research on tension control must begin with an nivestigation of tensio changes when draft or friction acts on a yarn.

There are a number of published reports in Japan on tension control. They include Maeda's theory [1,2, $3,4]$ which treats rheologically of the tension of viscose and nylon yarns in running state; and Sakai's $[5,6,7]$ analysis which assumes a yarn to be elastic. Both studies dealt with the tension of a yarn running between two cylinders.

The present article is based on experiments on a winding system described in what follows. It gives friction to a yarn at two points and drafts it in two zones between the rollers. The article looks in to the setp response and the steady-state response of tension in a running yarn.

\section{Method of Experiment}

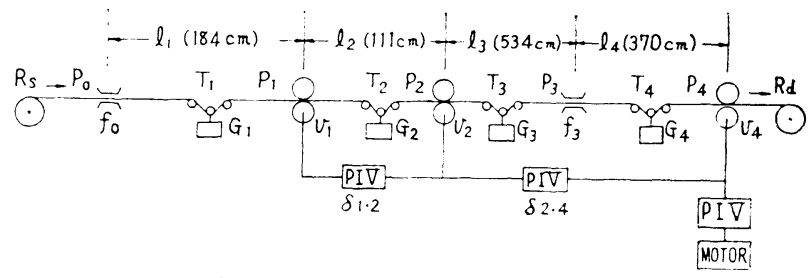

Fig. 1 experimental apparatus

One thread delivered from a yarn package (e.g., a bobbin or cone) was wound on bobbin $R_{d}$ through the winding system, as shown in Fig.1. The details follows:

\section{2-1 Yarn}

Three kinds of yarns, described by the following symbols, were used in our experiments:

$N_{1}$ : Nylon multifilament yarn (100 denier-2420) delivered from a bobbin on which it had been wound with a constant winding tension. 
$N_{2}$ : The same nylon as $N_{1}$ but used after being left in tensionless state for 72 hours.

$C$ : Cotton combed yarn $(1 / 10 \mathrm{~S})$ delivered from a cone on which it had been wound with a constant winding tension.

\section{2-2. Experimental Appratus}

The winding system used for this experiments is illustrated in Fig.1.

$R \mathrm{~s}$ in Fig. 1 is the area from which the yarn was delivered and where a yarn package was set up. $P_{0}$ is a tension device of disc type. $P_{1}, P_{2}$ and $P_{4}$ are rollers driven positively by a three-phase induction mortor through the medium of a variable-speed gear of PIV type. $P_{3}$ is a guide roller, driven passively by frictional force between the running yarn and the guide roller.

$R_{d}$ is the winding part where a running yarn was wound passively with the surface velocity of roller $P_{4}$.

$T_{1}, T_{2}, T_{3}$ and $T_{4}$ express the degrees of yarn tension in the zones between, respectively, $P_{0}$ and $P_{1}$, $P_{1}$ and $P_{2}, P_{2}$ and $P_{3}$ and $P_{3}$ and $P_{4}$. These tension degrees were measured with tensionmeters with strain gages attached. The tensionmeters are represented by $G_{1}, G_{2}, G_{3}$ and $G_{4}$.

We investigated experimentally the effects of the following five factors on tension in our system:

$f_{0}$ : Frictional force acting on a running yarn when a weight was put on $P_{0}$.

$\delta_{1.2}:$ Draft estimated from the difference in surface velocity between $P_{1}$ and $P_{2}$. The draft can be varied by the variable speed gear of PIV type placed between $P_{1}$ and $P_{2}$.

$\delta_{2 \cdot 4}$ : Draft estimated from the difference in surface velocity between roller $P_{2}$ and $P_{4}$.

$f_{3}$ : Frictional force acting on a running yarn when a brake was applied to guide roller $P_{3}$.

$V: \quad$ Yarn velocity defined by the surface velocity of roller $P_{4}$.

A yarn was delivered from $R_{s}$, ran through $P_{0}$, $P_{1}, P_{2}, P_{3}$ and $P_{4}$ in the winding system, and was wound in winding part $R_{d}$. Yarn tension between $R_{s}$ and $P_{0}$ was kept tensionless (nil). Then the yarn was kept in standard state. When the value of only one of these factors was varied stepwise, the responses of the various degrees of tension were measured.

The four other factors were kept in standard state, a state in which the values of factors $f_{0}, \delta_{1 \cdot 2}, \delta_{2 \cdot 4}$. $f_{3}$ and $V$ were, respectively, $1,0 \%, 1 \%, 0$ (zero) and 20 centimeters per second. $f_{0}=1$ means a unit of weight added to tension device $P_{0} ; f=2$ means two units of weight added. Draft $\delta_{1 \cdot 2}$ and draft $\delta_{2 \cdot 4}$ are expressed as values $\left(\nu_{2}-\nu_{1}\right) / \nu_{1}$ and $\left(\nu_{4}-\nu_{3}\right) / \nu_{2}$, respectively, where $\nu_{1}=$ surface velocity of $P_{1}, \nu_{2}=$ surface velocity of $P_{2}$, and $\nu_{4}(=V)=$ surface velociyt of $P_{4}$. The value of $f_{3}$ shows the number of the guide rollers to which a brake was applied. Yarn velocity $V$ in standard state was 20 centimeters per second.

The transient response of tension was measured at intervals of three minutes when yarn velocity was $V=20 \mathrm{~cm} / \mathrm{sec}$. The reason is that it took yarn tension three minutes to enter steady state after the variation of the value of the various factors stepwise for $V=20$ $\mathrm{cm} / \mathrm{sec}$. Therefore, the value after three minutes for $V=20 \mathrm{~cm} / \mathrm{sec}$ was used as the steady-state value of yarn tension.

\section{Theoretical Analysis}

\section{3-1. Transfer Function}

Assuming a yarn to be perfectly elastic, the tension of a running yarn is calculable theoretically thus, the following symbols being used:

$l_{i}: \quad$ Distance between $P_{i-2}$ and $P_{i}$ where $i=1,2$, 3,4 .

$\rho_{i}$ : Linear density of yarn between $P_{i-1}$ and $P_{i}$.

$\tau$ : $\quad$ Elastic modulus of yarn

$\nu_{1}, \nu_{2}, \nu_{4}:$ Surface velocities of rollers $P_{1}, P_{2}$ and $P_{4}$, respectively.

$\left[T_{1}\right]$ Assuming that the friction and the inertia in delivery part $R_{\mathrm{S}}$ is negligible, we obtain the following approximate equation:

$$
T_{1}=f_{0}
$$

where $\mathrm{nj}$ : frictional force given to the yarn at nj $\left[T_{2}\right]$ Equation of continuity between $P_{1}$ and $P_{2}$ is

$$
l_{2} \frac{d \rho_{2}}{d t}=v_{1} \rho_{1}-v_{2} \rho_{2}
$$


Applying Hook's Law, we obtain:

$$
\rho_{0} / \rho_{i}=1+T_{i} \gamma \quad(i=1,2,3,4)
$$

where $\rho_{0}$ : value of the linear density of yarn between $R_{S}$ and $P_{0}$.

when $T_{i} \rightarrow T_{i}+\Delta T_{i}$ and $\rho_{i} \rightarrow \rho_{i}+\Delta \rho_{i}$, from eq.(3) emerges eq.(4):

$$
\frac{\rho_{i}}{\rho_{i}+\Delta \rho_{i}}=\frac{1+T_{i} / \gamma+\Delta T_{i} / \gamma}{1+T_{i} / \gamma}
$$

Here, $\Delta T_{i}$ and $\Delta \rho_{i}$ are much smaller than $T_{i}$ and $\rho_{i}$. Assuming $T_{i} \ll \tau$, the following relation is deducible from eq.(4):

$$
\Delta T_{i} \fallingdotseq-\gamma \frac{\Delta \rho_{i}}{\rho_{i}}
$$

Assuming $\bar{\nu}_{1}, \bar{\nu}_{2}, \bar{\rho}_{1}$ and assuming $\bar{\rho}_{2}$ to be constant and $\Delta \nu_{1}, \Delta \nu_{2}, \Delta \rho_{1}$, and $\Delta \rho_{2}$ variable, we obtain $v_{1}=\bar{v}_{1}+\Delta v_{1}, v_{2}=\bar{v}_{2}+\Delta v_{2}, \rho_{1}=\bar{\rho}_{1}+\Delta \rho_{1} \quad \rho_{2}=\overline{\rho_{2}}+\Delta \rho_{2}$.

Therefore:

$$
\frac{d \bar{\rho}_{2}}{d t}=\frac{d\left(\rho_{2}+\Delta \rho_{2}\right)}{d t}=\frac{d \Delta \rho_{2}}{d t}
$$

and $\bar{v}_{1} \bar{\rho}_{1}-\bar{v}_{2} \bar{\rho}_{2}=0$

If we ignore the second order infinitesimal, then:

$$
\begin{aligned}
v_{1} \rho_{1}-v_{2} \rho_{2}= & \left(\bar{v}_{1}+\Delta v_{1}\right)\left(\bar{\rho}_{1}+\Delta \rho_{1}\right) \\
& -\left(\bar{v}_{2}+\Delta v_{2}\right)\left(\bar{\rho}_{2}+\Delta \rho_{2}\right) \\
= & \bar{v}_{1} \Delta \rho_{1}+\bar{\rho}_{1} \Delta v_{1}-\bar{v}_{2} \Delta \rho_{2}-\bar{\rho}_{2} \Delta v_{2}
\end{aligned}
$$

Accordingly, eq.(2) transforms as follows:

$$
l_{2} \frac{d \Delta \rho_{2}}{d t}=\bar{v}_{1} \Delta \rho_{1}+\bar{\rho}_{1} \Delta v_{1}-\bar{v}_{2} \Delta \rho_{2}-\bar{\rho}_{2} \Delta v_{2}
$$

Rewrite eq.(5) thus: $\rho_{i}=\overline{\rho_{i}}$. Then from eqs.(5) and (6) emerges this other equation:

$$
\frac{l_{2}}{\bar{v}_{2}} \cdot \frac{d \Delta T_{2}}{d t}+\Delta T_{2}=\Delta T_{1}+\gamma\left(\frac{\Delta v_{2}}{v_{2}}-\frac{\Delta v_{1}}{v_{1}}\right)
$$

which, by Laplace Transform, is expressible as follows:

$$
L\left[\Delta T_{2}\right]=\frac{1}{1+s l_{2} / v_{2}}\left\{L\left[\Delta T_{1}\right]+\gamma L\left[\frac{\Delta v_{2}}{\bar{v}_{2}}-\frac{\Delta v_{1}}{\bar{v}_{1}}\right]\right\}
$$

$$
\text { Since } \begin{aligned}
\Delta \delta_{1 \cdot 2} & =\Delta\left(\frac{v_{2}-v_{1}}{v_{1}}\right), \\
\Delta \delta_{1 \cdot 2} & =\frac{\Delta v_{2}-\Delta v_{1}}{\bar{v}_{1}}-\frac{\bar{v}_{2}-\bar{v}_{1}}{\bar{v}_{1}^{2}} \cdot \Delta v_{1} \\
& =\frac{\Delta v_{2}-\Delta v_{1}}{\bar{v}_{1}}-\delta_{1 \cdot 2} \frac{\Delta v_{1}}{\bar{v}_{1}}
\end{aligned}
$$

$\nu_{1}$ being approximately equal in value to $\bar{\nu}_{2}$ and $\delta_{1.2}$ being small in value, we obtain:

$$
\Delta \delta_{1 \cdot 2} \fallingdotseq \frac{\Delta v_{2}-\Delta v_{1}}{\bar{v}_{1}} \fallingdotseq \frac{\Delta v_{2}}{\bar{v}_{2}}-\frac{\Delta v_{1}}{\bar{v}_{1}}
$$

Substituting this equation into eq.(7) gives us:

$$
L\left[\Delta T_{2}\right]=\frac{1}{1+s t_{2}}\left\{L\left[\Delta T_{1}\right]+\gamma L\left[\Delta \delta_{1 \cdot 2}\right]\right\}
$$

$$
\text { where } \quad l_{2} / v_{2}=t_{2}\left(l_{2} / V\right)
$$

$\left[T_{3}, T_{4}\right]$ Equation of continuity between $P_{2}$ and $P_{4}$ is given by this equation:

$$
l_{3} \frac{d \rho_{3}}{d t}+l_{4} \frac{d \rho_{4}}{d t}=v_{2} \rho_{2}-v_{4} \rho_{4}
$$

The relation between frictional force $f_{3}$ generated when a running yarn drives the guide roller and tension degrees $T_{3}$ and $T_{4}$ is expressible approximately as follows: 


$$
T_{4}-f_{3}=T_{3}
$$

Therefore, in the same way in which eq.(8) is obtained, these two equations are obtainable from the foregoing two:

$$
\begin{aligned}
L\left[\Delta T_{3}\right]= & \frac{1}{1+s\left(t_{3}+t_{4}\right)}\left\{L\left[\Delta T_{2}\right]\right. \\
& \left.-\left(1+s t_{4}\right) L\left[\Delta f_{3}\right]+\gamma L\left[\Delta \delta_{2.4}\right]\right\}
\end{aligned}
$$

$$
\begin{aligned}
L\left[\Delta T_{4}\right]= & \frac{1}{1+s\left(t_{3}+t_{4}\right)}\left\{L\left[\Delta T_{2}\right]\right. \\
& \left.+s t_{3} L\left[\Delta f_{3}\right]+\gamma L\left[\Delta \delta_{2 \cdot 4}\right]\right\}
\end{aligned}
$$

\begin{tabular}{|c|c|c|c|c|c|}
\hline in put & 1 & $\Delta T_{1}$ & $\Delta T_{2}$ & $\Delta T_{3}$ & $T \Delta_{4}$ \\
\hline \multirow{3}{*}{$\Delta f_{0}$} & $\begin{array}{l}\text { Transfer } \\
\text { function }\end{array}$ & 1 & $\frac{1}{1+s t_{2}}$ & $\frac{1}{\left(1+s t_{2}\right)\left\{1+s\left(t_{3}+t_{4}\right)\right\}}$ & Same as $\Delta T_{3}$ \\
\hline & Step response & 1 & $1-e^{-t / t_{2}}$ & $1-\frac{t_{3}+t_{4}}{t_{3}+t_{4}-t_{2}} e^{-\frac{t}{t_{3}+t_{4}}}+\frac{t_{2}}{t_{3}+t_{4}-t_{2}} e^{-t / t_{2}}$ & Same as $\Delta T_{3}$ \\
\hline & $\begin{array}{l}\text { Steady-state } \\
\text { response }\end{array}$ & 1 & 1 & 1 & 1 \\
\hline \multirow{3}{*}{$\Delta \delta_{1 \cdot 2}$} & $\begin{array}{l}\text { Transfer } \\
\text { function }\end{array}$ & - & $\frac{\gamma}{1+s t_{2}}$ & $\frac{\gamma}{\left(1+s t_{2}\right)\left\{1+s\left(t_{3}+t_{4}\right)\right\}}$ & Same as $\Delta T_{3}$ \\
\hline & Step response & & $\gamma\left(1-e^{-t / t_{2}}\right)$ & $\gamma\left\{1-\frac{t_{3}+t_{4}}{t_{3}+t_{4}-t_{2}} e^{-\frac{t}{t_{3}+t_{4}}}+\frac{t_{2}}{t_{3}+t_{4}-t_{2}} e^{-t / t_{2}}\right.$ & Same as $\Delta T_{3}$ \\
\hline & $\begin{array}{l}\text { Steady-state } \\
\text { response }\end{array}$ & 0 & $\gamma$ & $\gamma$ & $\gamma$ \\
\hline \multirow{3}{*}{$\Delta \delta_{2 \cdot 4}$} & $\begin{array}{l}\text { Transfer } \\
\text { function }\end{array}$ & - & - & $\frac{\gamma}{1+s\left(t_{3}+t_{4}\right)}$ & Same as $\Delta T_{3}$ \\
\hline & Step response & - & - & $\gamma\left(1-e^{-\frac{t}{t_{3}+t_{4}}}\right)$ & Same as $\Delta T_{3}$ \\
\hline & $\begin{array}{l}\text { Steady-state } \\
\text { response }\end{array}$ & 0 & 0 & $\gamma$ & $\gamma$ \\
\hline \multirow{3}{*}{$\Delta f_{3}$} & $\begin{array}{l}\text { Transfer } \\
\text { function }\end{array}$ & - & - & $-\frac{1+s t_{4}}{1+s\left(t_{3}+t_{4}\right)}$ & $\frac{s t_{3}}{1+s\left(t_{3}+t_{4}\right)}$ \\
\hline & Step response & - & 一 & $\frac{t_{3}}{t_{3}+t_{4}} e^{-\frac{t}{t_{3}+t_{4}}}-1$ & $\frac{t_{3}}{t_{3}+t_{4}} e^{-\frac{t}{t_{3}+t_{4}}}$ \\
\hline & $\begin{array}{l}\text { Steady-state } \\
\text { response }\end{array}$ & 0 & 0 & -1 & 0 \\
\hline
\end{tabular}

$$
\text { where } \quad t_{3}=l_{3} / v_{3}\left(\fallingdotseq l_{3} / V\right), t_{4}=l_{4} / v_{4}\left(\fallingdotseq l_{3} / V\right)
$$

from which we deduce the transfer function shown in Table 1.

Table 1 Theoretical Responses of Tension 

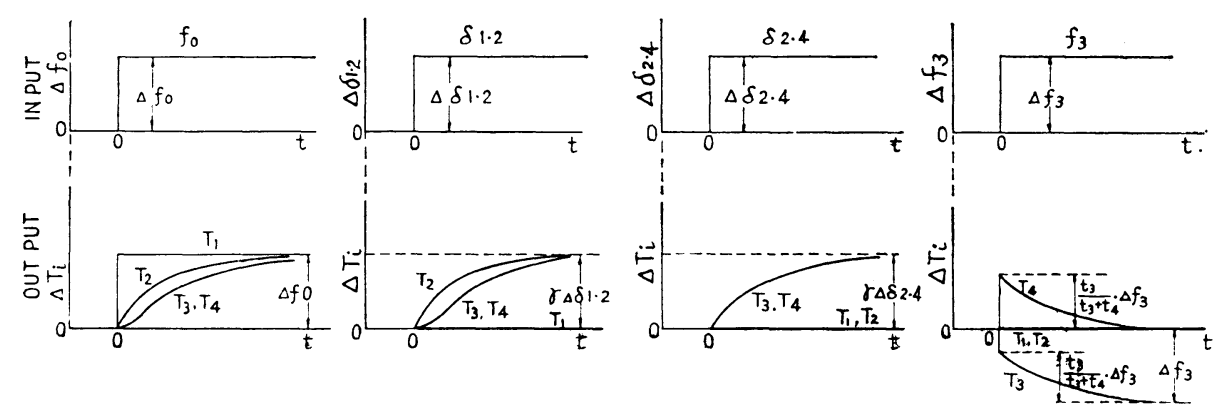

Fig. 2 Theoretical step response

\section{3-2. Transient Performance (step response)}

The step responses of tension in a running yarn given friction or drafted stepwise are calculable by the above theory with the results shown in Fig. 2 and Table 1. In other words, tension $T_{1}$, when given frictional force $\Delta f_{0}$ stepwise, increases approximately stepwise by $\Delta T_{1}$, the step response of $T_{2}$ being the first order lag and the step responses of $T_{3}$ and $T_{4}$ the second order lag.

When a running yarn is drafted stepwise by $\Delta \delta_{1.2}$, the step response of $T_{2}$ is the first order lag; those of $T_{3}$ and $T_{4}$, the second order lag. However, tension $T_{1}$ is not affected by draft. When a running yarn is drafted stepwise by $\Delta \delta_{2.4}$, the step responses of $T_{3}$ and $T_{4}$ are the first order lag. Neither $T_{1}$ nor $T_{2}$ is affected by draft $\delta_{2.4}$.

When a running yarn is given frictional force $\Delta f_{3}$ stepwise, tension $T_{4}$ increases by $\left(t_{3} / t_{3}+t_{4}\right) \Delta f_{3}$ instantaneously, and thereafter, decreases exponentially with time as shown in Fig. 2. Tension $T_{3}$ decreases by $\left(t_{4} / t_{3}+t_{4}\right) \Delta f_{3}$ instantaneously and thereafter decreases exponentially with time.

3-3. Steady-state Performance (Steady-state response)

Tensions in steady state are expressible in value as follows:

$$
\left[T_{1}\right] \quad T_{1}=f_{0}
$$

[ $\left.T_{2}\right]$ From eq.(3) emerges this equation:

$$
\rho_{1} / \rho_{2}=\left(\gamma+T_{2}\right) /\left(\gamma+T_{1}\right),
$$

and as $\rho_{1} / \rho_{2}=v_{2} / v_{1}=1+\delta_{1 \cdot 2}$, we obtain:

$$
\begin{aligned}
T_{2} & =T_{1}\left(1+\delta_{1 \cdot 2}\right)+\gamma \delta_{1 \cdot 2} \quad \delta_{1 \cdot 2} \ll 1 \\
& =T_{1}+\gamma \delta_{1 \cdot 2}
\end{aligned}
$$

Substituting eq.(11) into the preceding equation leads to:

$$
T_{2}=f_{0}+\gamma \delta_{1 \cdot 2}
$$

$$
\left[T_{3}, T_{4}\right] \text { Similarly, we obtain: }
$$

$$
\begin{aligned}
T_{4} & =T_{2}\left(1+\delta_{2 \cdot 4}\right)+\gamma \delta_{2 \cdot 4} \quad \gamma_{2 \cdot 4} \ll 1 \\
& \fallingdotseq T_{2}+\gamma \delta_{2 \cdot 4} \\
& =f_{0}+\gamma\left(\delta_{1 \cdot 2}+\delta_{2 \cdot 4}\right) \\
& =f_{0}+\gamma \delta_{1 \cdot 4}
\end{aligned}
$$

where $\quad \delta_{1 \cdot 4}=\frac{v_{4}-v_{1}}{v_{1}} \fallingdotseq \frac{v_{2}-v_{1}}{v_{1}}+\frac{v_{4}-v_{2}}{v_{2}}=\delta_{1.2}+\delta_{2.4}$ gives us:

Substituting equation, $T_{3}=T_{4}-f_{3}$, into eq.(13)

$$
T_{3}=f_{0}+\gamma \delta_{1.4}-f_{3}
$$

\section{4-1. Transient Response}

Five factors (i.e., $f_{0}, \delta_{1 \cdot 2}, \delta_{2 \cdot 4}, f_{3}$ and $V$ ) were varied stepwise one by one and their effects on tension in each zone were examined as shown in Fig.3.

Except one factor changed stepwise, the four other factors were kept in standard state as mentioned above, i.e., $f_{0}=1, \delta_{1 \cdot 2}=0 \%, \delta_{2 \cdot 4}=1 \%, f_{3}=0$ and $V=20$ $\mathrm{cm} / \mathrm{sec}$ Fig.3a shows the step responses of various degrees of tension measured when the value of $f_{0}$ was varied stepwise from 0 to 2 . Similarly, Figs.3b, $3 \mathrm{c}$ and $3 \mathrm{~d}$ show the step responses of various degrees of tension measured when $\delta_{1 \cdot 2}, \delta_{2 \cdot 4}$ and $f_{3}$ were varied in value from $0.5 \%$ to $0.5 \%$, from $0.5 \%$ to $1.5 \%$ and from zero to one 


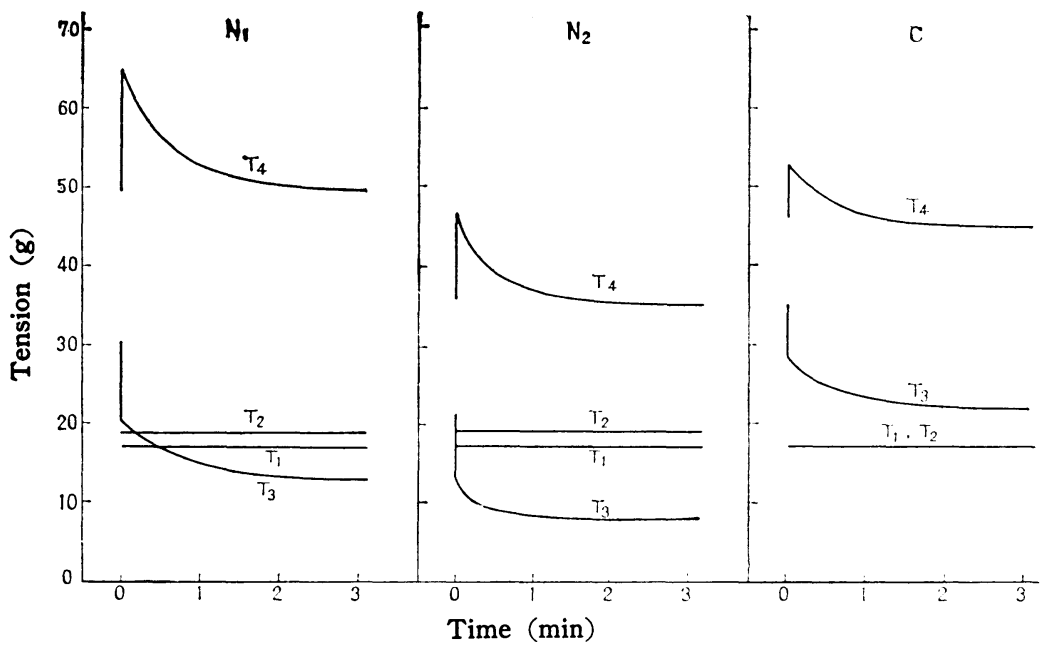

Fig. 3d Step response $\left(f_{3}=0 \rightarrow 1\right)$

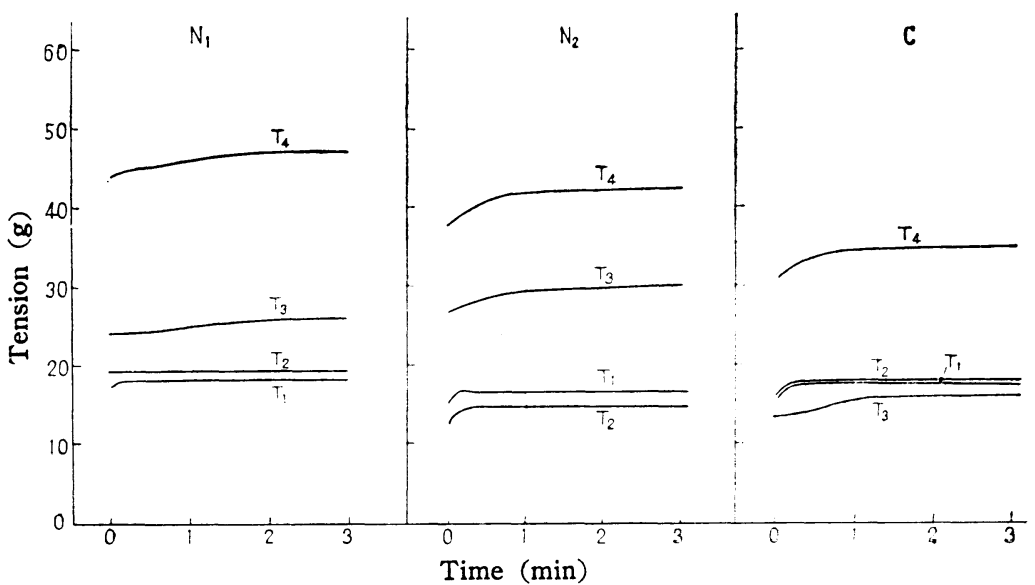

Fig. 3e Behavior of tension $(V=10 \mathrm{~cm} / \mathrm{sec} \rightarrow 20 \mathrm{~cm} / \mathrm{sec})$

Fig. 3 Experimental step response perimental results, especially for sample $N_{2}$, as shown in Table 2 .

In steady state, $T_{2}-T_{1}=\tau \delta_{1.2}$ and $. T_{4}-T_{2}=\tau \delta_{2.4}$. Therefore, the relation between $T_{2}-T_{1}$ and $\delta_{1.2}$ was linear as a result of the experiment like the relation between $T_{4}-T_{2}$ and $\tau \delta_{2 \cdot 4}$ as shown in Figs. $4 b$ and $4 c$.

For these relations to accord more exactly to the theory, the slope in Fig.4b must be the same as the slope in Fig.4c. However, the elastic modulus estimated from the slope in Fig.4b was larger than that estimated from Fig. $4 c$, as shown in Table 3. The values of $l_{2}$ and $l_{3}+$ $l_{4}$ (ie.,distance between $P_{2}$ and $P_{4}$ were 1.11 and 9.04 meters. Accordingly, the time required for a yarn to run from $P_{1}$ to $P_{2}$ is shorter than the time needed for a run from $P_{2}$ to $P_{4}$.

Maeda [1] defined the time which required for a yarn to run from a delivery roller to a winding roller as creep time and said the shorter this time, the heavier the yarn tension. The elastic modulus estimated from

Table 2 Time Constant

\begin{tabular}{|c|c|c|c|c|c|c|c|c|c|c|c|c|c|c|c|c|}
\hline \multirow[b]{3}{*}{ Foctors } & \multicolumn{12}{|c|}{ Experimental results } & \multirow{2}{*}{\multicolumn{4}{|c|}{ Theoretical values }} \\
\hline & \multicolumn{4}{|c|}{$N_{1}$} & \multicolumn{4}{|c|}{$N_{2}$} & \multicolumn{4}{|c|}{$C$} & & & & \\
\hline & $T_{1}$ & $T_{2}$ & $T_{3}$ & $T_{4}$ & $T_{1}$ & $T_{2}$ & $T_{3}$ & $T_{4}$ & $T_{1}$ & $T_{2}$ & $T_{3}$ & $T_{4}$ & $T_{1}$ & $T_{2}$ & $T_{8}$ & $T_{4}$ \\
\hline$\underset{0 \rightarrow 2}{f_{0}}$ & 0 & 7 & 61 & 61 & 0 & 4 & 68 & 55 & 0 & 6 & 42 & 42 & 0 & 5.6 & 51.2 & 51.2 \\
\hline$\underset{-0.5 \rightarrow 0.5 \%}{\delta_{1 \cdot 2}}$ & & 8 & 54 & 52 & & 6 & 50 & 40 & & 7 & 46 & 39 & & 5.6 & 51.2 & 51.2 \\
\hline$\underset{0.5 \rightarrow 1.5 \%}{\delta_{2 \cdot 4}}$ & & & 51 & 49 & & & 28 & 25 & & & 43 & 42 & & & 45.2 & 45.2 \\
\hline$\underset{0 \rightarrow 1}{f_{3}}$ & & & 40 & 35 & & & 12 & 25 & & & 40 & 38 & & & 45.2 & 45.2 \\
\hline
\end{tabular}




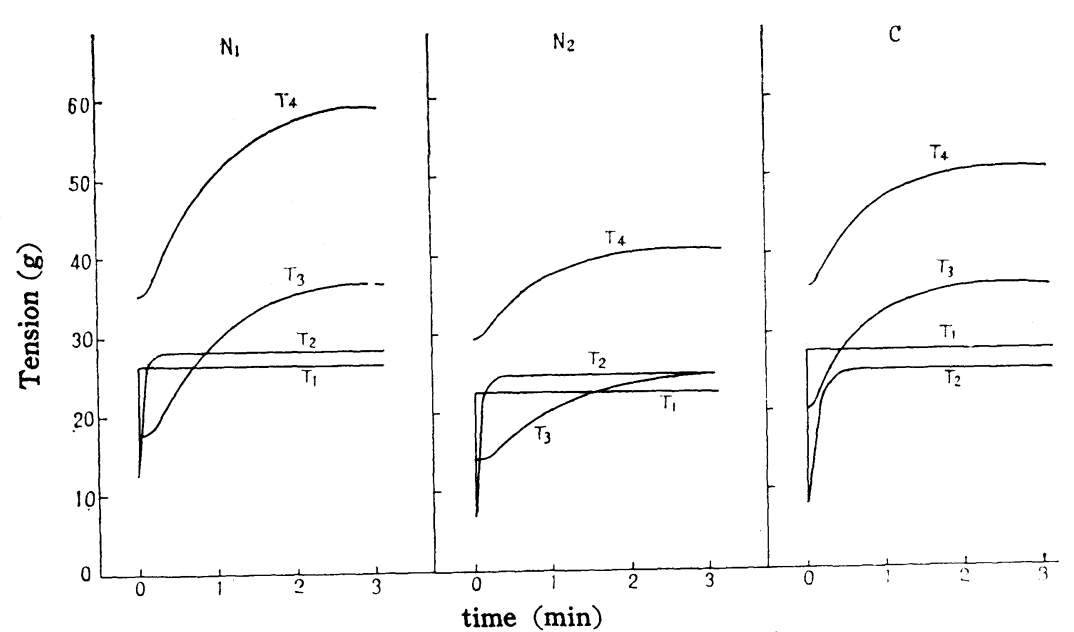

Fig. 3a Step response $\left(f_{0}=0 \rightarrow 1\right)$

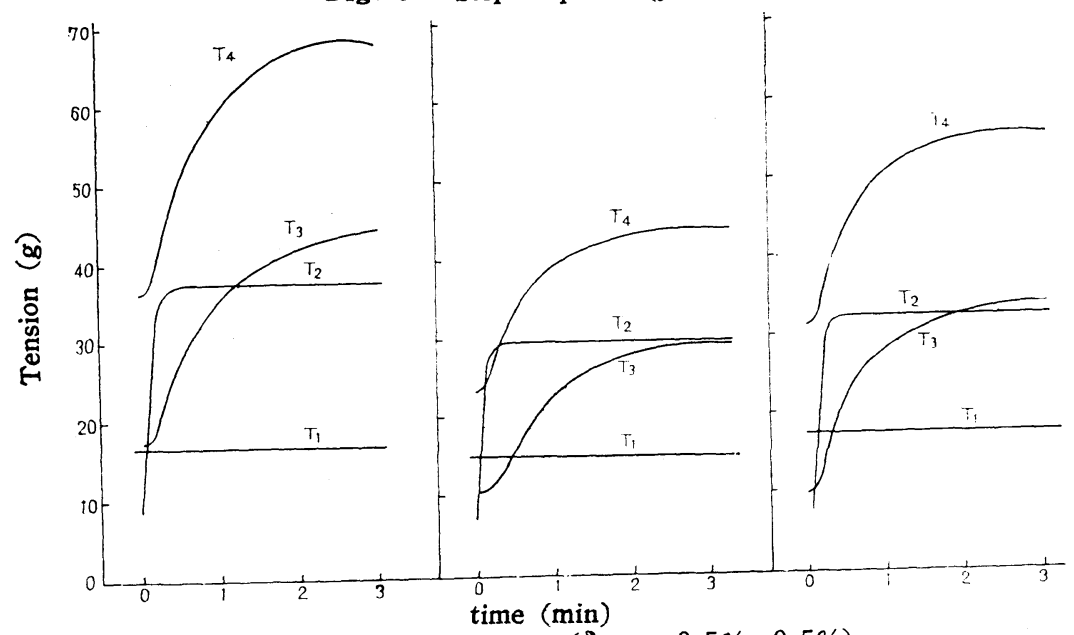

Fig. 3b Step response $\left(\delta_{1.2}=-0.5 \% \rightarrow 0.5 \%\right)$

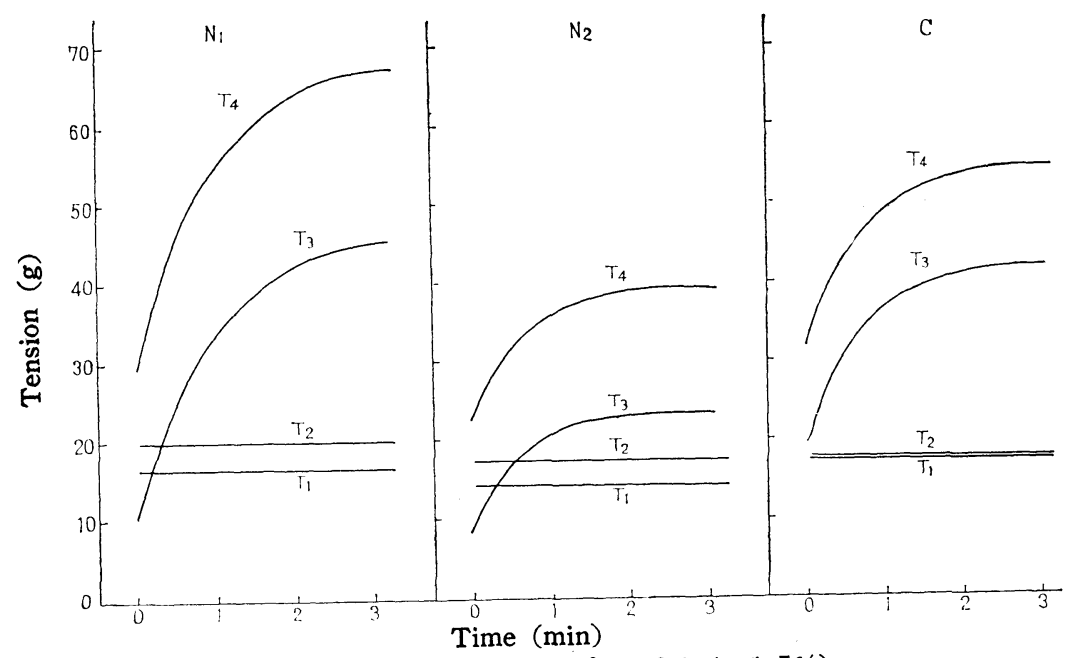

Fig. 3c Step response $\left(\delta_{2.4}=0.5 \% \rightarrow 1.5 \%\right)$

Fig. 3 Experimental step response
Table 2 gives a time constant estimated from the experimental results in Fig. 3 and calculated on the theory stated above.

4-2. Steady-state Response

According to the theory in steady state, the following equations emerge from eqs. (11), (12), (13) and (14):

$$
\begin{aligned}
& T_{1}=f_{0} \\
& T_{4}-T_{2}=\tau \delta_{1 \cdot 2} \\
& T_{4}-T_{2}=\tau \delta_{1}: 2 \\
& T_{4}-T_{3}=f_{3}
\end{aligned}
$$

Tension degrees $T_{1}, T_{2}, T_{3}$ and $T_{4}$, observed three minutes after these factors were varied stepwise, were used as steady-state values, the yarn velocity being fixed at $20 \mathrm{~cm} / \mathrm{sec}$. The values of $T_{1}$, $\left(T_{2}-T_{1}\right),\left(T_{4}-T_{2}\right)$ and $\left(T_{4}-\right.$ $T_{3}$ ) were plotted on the Y-axis. and the values of the factors were plotted on the $\mathrm{X}$-axis. These plots are given in Fig.4.

5-1. Effects of the Draft and Frictional Force.

Assuming a yarn to be perfectly elastic, the step responses of various degrees of tension on a running yarn given friction or draft are readily calculable and agreed qualitatively with the experimental results on nylon and cotton yarn. This is clear from a comparison of Fig.3 with Fig.2 or Table 1. In steady state, too, the experimental relation between tension and the factors was approximately linear, as shown in Fig.4 and, therefore, agreed qualitatively with the theory.

Quantitatively speaking, the theoretical values of various degrees of tension agreed approximately with the experimental results as to both step response and steady-state response. There were, however, some differences in time constant between theoretical values and ex- 


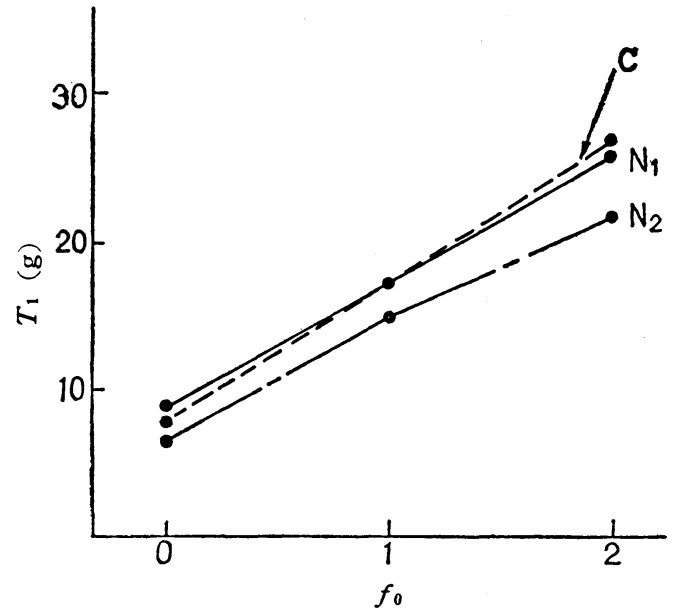

Fig. 4a Effect of $f_{0}$ on $T_{1}$

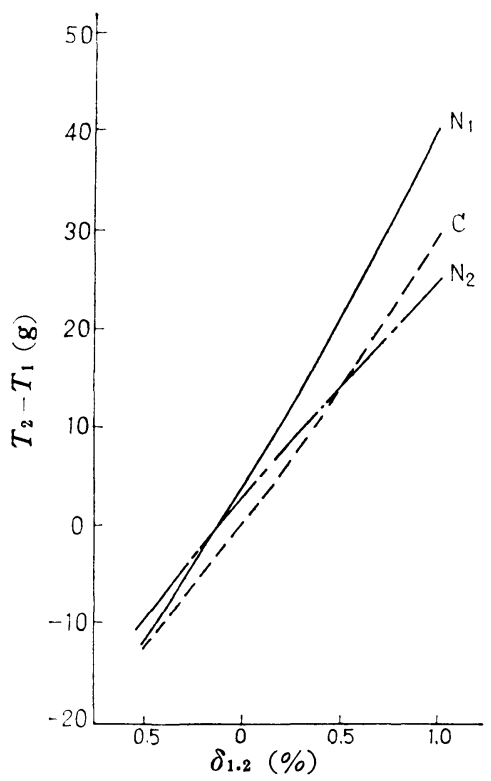

Fig. 4b Effect of $\delta_{1.2}$ on $\left(T_{2}-T_{1}\right)$

Fig. $4 \mathrm{~b}$ is larger than that estimated from Fig. $4 \mathrm{c}$, the difference being presumably explainable by the Maeda theory.

5-2. Effect of Yarn Velocity

It is believed that when yarn velocity increases, yarn tension does, too, because friction or inertia in the winding system increases and because a yarn takes less time to run between the delivery roller and the winding roller.

However, as shown in Fig. 3 e, tension in a running yarn in our experiments by only $15 \%$, at most, indicating only a slight effect of yarn velocity on yarn tension.

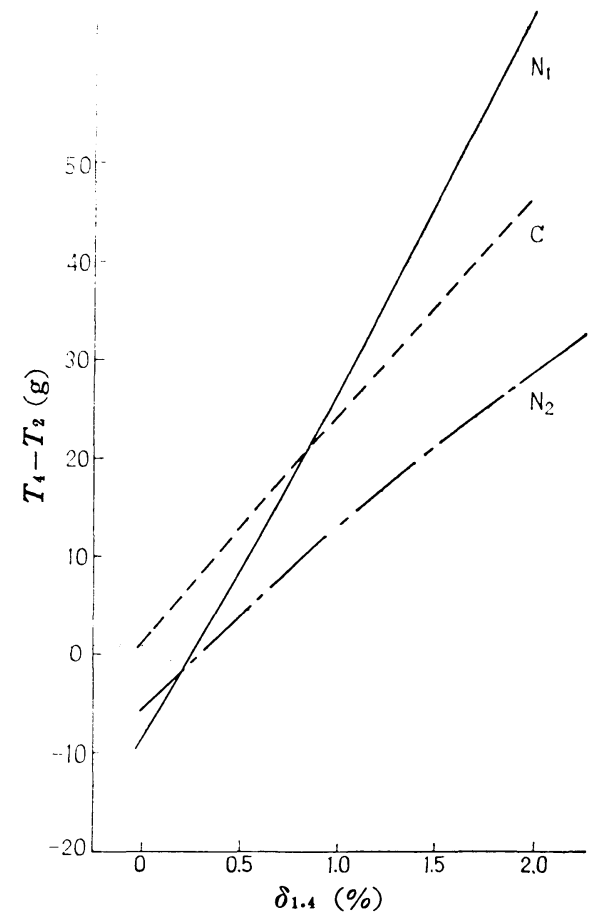

Fig. 4c Effect of $\delta_{2.4}$ on $\left(T_{4}-T_{2}\right)$

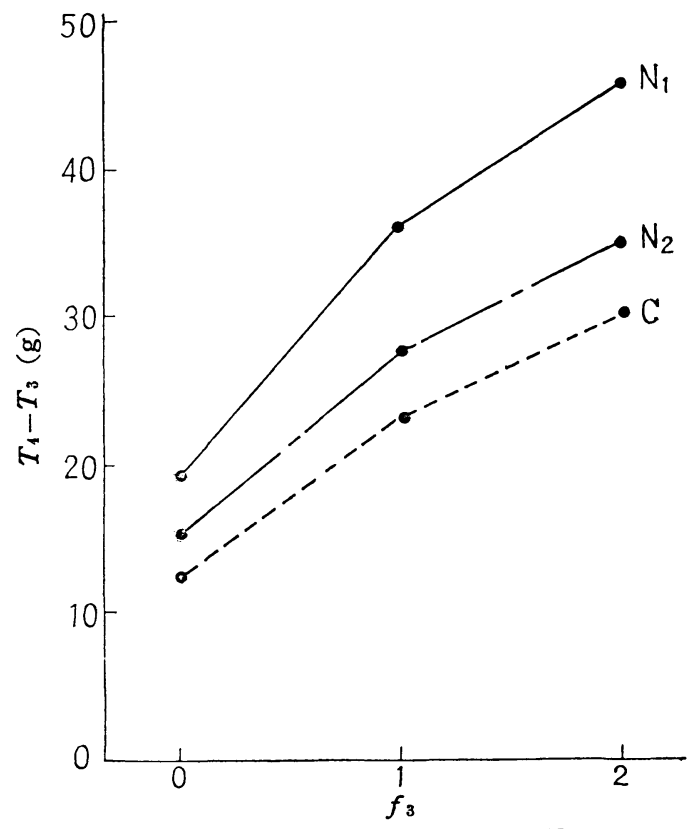

Fig. 4d Effect of $f_{3}$ on $T_{4}-T_{3}$

Fig. 4 Effects of factors on tension in steady state 
Table 3 Elastic Modulus $(\gamma)$

\begin{tabular}{l|c|c|c|c}
\hline \multicolumn{2}{c|}{ Measuring method } & $N_{1}$ & $N_{2}$ & $C$ \\
\hline $\begin{array}{l}\text { Values measured } \\
\text { by static stress- } \\
\text { strain curves }\end{array}$ & $\begin{array}{c}20^{\circ} \mathrm{C} \\
65 \% \mathrm{RH}\end{array}$ & 4.35 & 3.45 & 3.25 \\
\hline $\begin{array}{l}\text { Values estimated } \\
\text { from Figs. 5.b } \\
\text { and 5.c }\end{array}$ & $\delta_{1 \cdot 2}:\left(T_{2}-T_{1}\right)$ & 3.70 & 2.25 & 3.00 \\
\cline { 2 - 5 } & $\delta_{2 \cdot 4}:\left(T_{4}-T_{2}\right)$ & 3.55 & 1.85 & 2.35 \\
\hline
\end{tabular}

(unit : $\mathrm{kg} /$ thread)

\section{5-3. Effect to Tension Career}

There were some differences in the responses of the tension between smaples $N_{1}$ and $N_{2}$ as shown in Figs. 3 and 4, presumably due to differences in the amount of tension these yarns had received prior to our experiments. Sample $N_{1}$ had been wound at aconstant winding tension on a bobbin. Sample mj had been left in tension less state for 72 hours.

winding tension on a bobbin. Sample $N_{2}$ had been left in tension less state for 72 hours.

Therefore, $N_{2}$ was smaller in elastic modulus than $N_{1}$ while $N_{2}$ received a larger effect of creep than did $N_{1}$. The elastic moduli of $N_{1}$ and $N_{2}$ estimated from static stress-strain curves were 4.35 and 3.45 , respectively, as shown in Table 3. (The relation between elastic modulus and creep is dealt with in detail by E.Catsiff
[8]). It follows, then, that tension in a running yarn is considerably influenced by the career of tension and by creep.

Tension remaining in the cotton yarn wound on a corn being slight (as will be explained in part 3), we did not investigate the effect of the "career" of the tension - - how long it had been in the yarn and how the yarn had been subjected to the tension before our experiments.

\section{References}

[1] M. Maeda and T. Yamaguchi ; J. Text. Mach. Soc. Japan, Japanese ed., 12, 183 (1959)

[2] M. Maeda and T. Yamaguchi ; J. Text. Mach. Soc. Japan, Japanese ed., 12, 493 (1959)

[3] M. Maeda and T. Yamaguchi ; J. Text. Mach. Soc. Japan, Japanese ed., 13, 177(1960)

[4] M. Maeda and T. Yamaguchi ; J. Text. Mach. Soc. Japan, Japanese ed., 14, 124(1961)

[5] T. Sakai and E. Kuze ; J. Text. Mach. Soc. Japan, Japanese ed., 14, 243(1961)

[6] T. Sakai and E. Kuze ; J. Text. Mach. Soc. Japan, Japanese ed., 14, 250 (1961)

[7] T. Sakai and E. Kuze ; J. Text. Mach. Soc. Japan, Japanese ed., 15, 670 (1962)

[8] E. Catsiff, et. al.; Text. Res. J., 23, 808 (1953) 